
\title{
R Research Soure \\ Theoretical and experimental study on the effective area of water film and dust removal efficiency
}

hua guo

Hunan University of Science and Technology https://orcid.org/0000-0001-5349-9827

hai qiao Wang

Hunan University of Science and Technology

shi qiang Chen ( $\square$ shiqiangchen@hnust.edu.cn )

zhi rong Wu

Hunan University of Science and Technology

\section{Research}

Keywords: resonant string grille, wetting mechanism, capillary action, effective water film area, dust removal efficiency

Posted Date: May 11th, 2020

DOI: https://doi.org/10.21203/rs.3.rs-25376/v1

License: (c) (i) This work is licensed under a Creative Commons Attribution 4.0 International License.

Read Full License 


\title{
Journal Title
}

\section{Theoretical and experimental study on the effective area of water film and dust removal efficiency}

\author{
Guo Hua1,2, Wang Haiqiao1, Chen Shiqiang3,4*Wu Zhirong1 \\ 1School of Resource, Environment and Safety Engineering, Hunan University of Science and Technology, \\ Xiangtan, 411201, Hunan, China \\ 2School of Civil and Environmental Engineering, Hunan University of Science and Engineering, \\ Yongzhou 425199, Hunan, China \\ 3Hunan Provincial Engineering Techniques Research Centre of Mine Ventilation \& Dedusting Equipment, \\ Hunan University of Science and Technology, 411201, Hunan, China \\ 4Work Safety Key Lab on Prevention and Control of Gas and Roof Disasters for Southern Coal Mines, \\ Hunan University of Science and Technology, Xiangtan, 411201, Hunan, China \\ Supported by the 2017 Hunan Provincial Graduate Research Innovation Project of China (No. \\ CX2017B649), National Natural Science Foundation of China (No. 51774134), Hunan Provincial Natural \\ Science Foundation of China (No. 2019JJ60044), and the Excellent youth project of Hunan Provincial \\ Department of Education (No. 19B223) \\ *Corresponding author. E-mail: shiqiangchen@hnust.edu.cn
}

\begin{abstract}
We investigate the capture process of dust flow through a vibrating wire, water fog, and a water film to address the problem of excessively high exhaust dust concentrations in mine exhaust shafts according to the theory of liquid-solid flow and capillary film formation. Functional expressions of the thickness and height of dust-trapping water film are derived by using Young-Laplace equations and Navier-Stokes equations, respectively. The theoretical relationship between the effective water film area and dedusting efficiency on a vibrating wire is obtained.The dedusting efficiency of a resonant chord grid is measured experimentally. The results show that wire spacing plays a decisive role in water film formation. The instantaneous effective water film area of the vibrating wire grid is proportional to the dedusting efficiency. When the diameter distance ratio of the resonance chord grid was 1.14 with the dedusting wind speed controlled at $3 \mathrm{~m} / \mathrm{s}$ and a spray pressure of $0.7 \mathrm{MPa}$. The total dust control efficiency can reach $>92 \%$.
\end{abstract}

Keywords:resonant string grille; wetting mechanism;capillary action;effective water film area; dust removal efficiency

\section{Introduction}

When dusty air flows through a resonant chord grid, a component collides with the chord grid and the rest passes through the chord grid's gap. The water film that forms between the chord grids plays an important role in dust removal. During airflow, droplets ejected from the 
nozzle cause a water film to continuously form and break on the chord grid. The broken water film coagulates from the presence of new droplets. With increasing coagulation volume, gravity increases, motion accelerates, and water slips along the growth rate of the vibrating wire. During this process, the formation and fragmentation of a water film and its effective area directly affect the dedusting efficiency of the vibrating wire grid plates.

From a theoretical and experimental perspective, Fairs [1] discovered the existence of a liquid film by studying the formation of a sulfuric acid vinegar fog on four kinds of fibers with different properties. Using a continuous spray of water mist on the fiber, Agranovski [2] designed a method to form a water film after a few minutes. Theoretical and experimental studies show that the water film thickness increases with fiber equivalent diameter and differs along different heights of the fiber grid. In the case of equilibrium saturation, Jakub [3,4] and Ryan [5] observed uneven liquid coverage on the fiber and studied the efficiency of filtertrapping aerosol. Gong [6] studied the dominant mechanism of water film formation on the fiber grid and pointed out the capillary effect of the fiber spacing. Jiang [7] and Jin [8] theoretically analyzed the dedusting mechanism of water films and assumed that dust particles that are difficult to moisten tend to penetrate into the water film. $\mathrm{Li}$ [9] and $\mathrm{Wu}$ [10] indirectly explained dust removal efficiency from the aspect of fiber grid gap, material, and grid, as well as nozzle spacing according to water films observed in experiments. However, most of these studies analyzed the existence of water films from a macro and indirect perspective and there remains a lack of systematic analysis of the physical mechanism in water film formation and fragmentation processes, which makes the relationship between water film state and dust removal efficiency poorly understood.

During wet resonant chord grid dedusting, the water film between the vibrating wire directly affects dedusting efficiency and resistance. Quantification of how the water film affects dust removal efficiency requires information regarding the interaction between the vibrating wire structure and fog droplets. In this study, we perform experiments and theoretical analyses of the dynamic water film dedusting process using a vibrating wire grid plate. The state of the water film on the vibrating wire grid plate is monitored during the experiments using a highspeed camera, and the influence of spray pressure, wind speed, and vibrating wire grid structure on the effective area of the transient water film is analyzed. A mathematical expression of effective water film area and dedusting efficiency is established according to the experimental results, which provides a reference for theoretical and engineering applications of resonant chord grid water film dedusting.

\section{Materials and Methods}

\section{Water film formation mechanism}

The water mist contacts the two adjacent wires and forms a water film owing to surface adhesion forces and capillary action. The Bond number is used to describe the capillary phenomenon in surface mechanics [15] and is the relative magnitude of static water mist gravity and surface tension:

$$
B o=\frac{\rho g \lambda_{c}{ }^{2}}{\delta_{l v}}
$$


where $\lambda_{c}$ is capillary length, which is considered here to be half of the vibrating wire spacing $d_{0}(\mathrm{~mm}), \rho$ is the water mist density $\left(\mathrm{kg} / \mathrm{m}^{3}\right), g$ is gravitational acceleration $(\mathrm{N} / \mathrm{kg})$, and $\delta_{l v}$ is the liquid gas surface tension $(\mathrm{N} / \mathrm{m})$. When the Bond number is large, surface tension produced by static water mist under capillary pressure can be ignored. A small Bond number $(<0.1)$ shows that the surface tension strong affects the hydrostatic gravity.

In the film formation test, the water is $20{ }^{\circ} \mathrm{C}, \delta_{l v}=7.3 \times 10^{-3} \mathrm{~N} / \mathrm{m}, g=9.8 \mathrm{~N} / \mathrm{kg}, \rho=994 \mathrm{~g} / \mathrm{m}^{3}$, combined with reasonable reference values [16] and $B o=0.0036$, then:

$$
\begin{aligned}
2 \lambda_{c} \leq 2\left(\frac{3.6 \times 10^{-3} \delta_{l v}}{\rho g}\right)^{\frac{1}{2}} \\
2 \lambda_{c} \leq 2\left(\frac{3.6 \times 10^{-3} \times 73 \times 10^{-3}}{994 \times 9.8} \times 10^{6}\right)^{\frac{1}{2}}=0.34 \mathrm{~mm}
\end{aligned}
$$

The distance between the vibrating wires is calculated from the equations above to be less than $0.34 \mathrm{~mm}$. The water mist generates a capillary phenomenon between the vibrating wires, which is consistent with the experimental results.

\section{Basic physical parameters of the chord gate}

The diameter of the vibrating wire is a particularly important physical parameter. The vibrating wire is cylindrical in shape and uniformly distributed in the wire grid with equal spacing. The diameter and spacing of the resonant chord gate (Table 1) are selected to facilitate the formation of a capillary water film on the gate and improve the dust removal efficiency of the resonant chord gate,.

Table 1 Dimensions of resonant string grille

\begin{tabular}{ccccc}
\hline Material & Filter area $(\mathrm{H} / \mathrm{mm} \times \mathrm{W} / \mathrm{mm}) / \mathrm{mm}^{2}$ & Layer number & $\begin{array}{c}\text { Vibrating wire } \\
\text { diameter } / \mathrm{mm}\end{array}$ & $\begin{array}{c}\text { Vibrating wire } \\
\text { spacing } / \mathrm{mm}\end{array}$ \\
Steel wire & $590 \times 550$ & 2 & 0.35 & 0.40 \\
Steel wire & $590 \times 550$ & 2 & & 0.65 \\
Steel wire & $590 \times 550$ & 2 & 0.29 & 0.21 \\
Steel wire & $590 \times 550$ & 2 & 0.23 & 0.46 \\
Steel wire & $590 \times 550$ & 2 & 0.17 & 0.27 \\
Steel wire & $590 \times 550$ & 2 & & 0.33 \\
\hline
\end{tabular}

According to analysis of the filtering mechanism of the resonant string grid, the vibrating wire is uniformly distributed on the rectangular chord grid and parallel to the airflow direction. The percentage of the steel wire area to the whole vibrating wire grid area prior to wetting is expressed by $\beta \mathrm{s}$. The wire-filling rate is determined during manual production and can be calculated according to Eq. (4). Voidage $\varepsilon s$ is obtained according to Eq. (5). The calculated results are listed in Table 2 .

$$
\begin{gathered}
\beta_{s}=\frac{\text { Area of vibrating wire }}{\text { Total area of grid plate }} \times 100 \% \\
\varepsilon_{s}=1-\beta_{s}
\end{gathered}
$$

Table 2 Filling rate and voidage of the resonant string grille

\begin{tabular}{cccccc}
\hline Material & Filter area $/ \mathrm{mm}^{2}$ & $\begin{array}{c}\text { Vibrating wire } \\
\text { diamete/mm }\end{array}$ & $\begin{array}{c}\text { Vibrating wire } \\
\text { spacing } / \mathrm{mm}\end{array}$ & $\begin{array}{c}\text { Filling rate } / \% \\
\text { Voidage } / \%\end{array}$ \\
Steel wire & $590 \times 550$ & 0.35 & 0.40 & 46.70 & 53.30 \\
Steel wire & $590 \times 550$ & & 0.65 & 35.00 & 65.00 \\
Steel wire & $590 \times 550$ & 0.29 & 0.21 & 58.00 & 42.00 \\
Steel wire & $590 \times 550$ & 0.23 & 0.46 & 38.67 & 61.33 \\
Steel wire & $590 \times 550$ & 0.17 & 0.37 & 46.00 & 54.00 \\
Steel wire & $590 \times 550$ & & & 34.00 & 66.00 \\
\hline
\end{tabular}


When the airflow carries water mist through the resonant chord grid, the filling rate of the wet resonant wire includes the water mist attached to the vibrating wire and the residual water film after the film ruptures. The filling rate of the chord grid is therefore composed of the filling rates of the unwetted wire and water:

$$
\begin{array}{r}
\beta_{w s}=\beta_{s}+\beta_{w} \\
\varepsilon=1-\beta
\end{array}
$$

where $\beta_{w s}$ and $\beta_{w}$ are the filling rates of the wire and water, respectively (dimensionless). The liquid holding capacity of the steel wire is measured and calculated experimentally [17] and $\varepsilon$ represents the gate voidage (dimensionless).

\section{Calculation of water film thickness and height on a vibrating wire grid plate}

(1)Water film thickness and height calculations

When the water mist and vibrating wire meet conditions of film formation, a liquid film force is produced, also known as a liquid bridge force [18].Capillary action theory can be used to analyze the liquid film force. The left and right sides of the water film between the vibrating string steel wires are connected owing to adhesion. On the other hand, there is surface tension on the upper and lower film surfaces, which forms a concave water film surface in the arc (Fig. 1) and leads to a pressure difference between the gas and body side of the water film (i.e. pressure outside the water film is greater than that inside). This negative pressure on the film itself has a pulling effect, which is essentially the liquid film force.

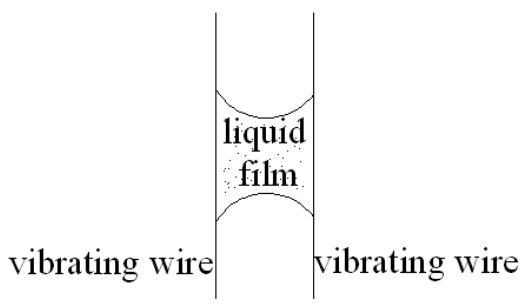

Fig. 1 Concave water film surface between vibrating wire strings

Derivation and calculation of water film thickness

During the tests, water mist is sprayed continuously on the vibrating wire grid plate with a small gap between the wires, and a water film forms under capillary action. The outer outline of the water film between the wires is a circular arc concave surface owing to the influence of the water film force (Fig. 2).

Let $d_{0}$ represent the distance between the two adjacent vibrating wires. The average curvature radius of the water film side surface is $r, r_{l}$ and $r_{2}$ are the curvature radius of the curved surface perpendicular and parallel to the steel wire, respectively, and the surface tension of the water film is $\delta$. According to the Young-Laplace [13] equation, the negative pressure difference of the water film between the vibrating wire is:

$$
\Delta p=\frac{2 \delta}{r}=\delta\left(\frac{1}{r_{1}}+\frac{1}{r_{2}}\right)
$$




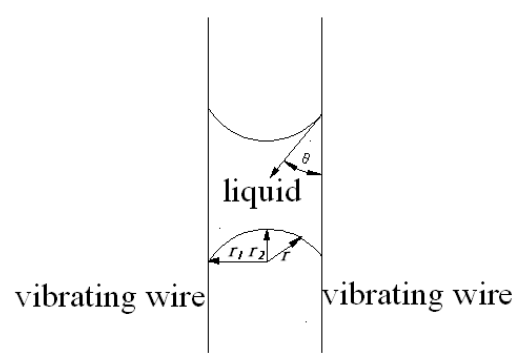

Fig. 2 Radius of the concave liquid surface

In a humid environment, when the water mist and parallel body of the wettable vibrating wire are close to one another, the vibrating surface absorbs the water mist molecules to form a water film. According to the physical and mechanical knowledge of surfaces and interfaces, the separation pressure $\mathrm{P} 0$ in the water film is expressed as:

$$
P_{0}=\frac{H}{6 \pi L^{3}}
$$

where $H$ is the Hamaker constant $(\mathrm{J})$ and $L$ is the water film thickness ( $\mathrm{mm}$ ).

Under mechanical equilibrium conditions, the negative pressure of the water film between the wires is equal to the separation pressure in the water film. Combined with the relationship between the curvature radius of the water film surface and relative humidity in the continuous spray environment, the Kelvin equation [19] shows:

$r_{k}=\left(\frac{1}{r_{1}}+\frac{1}{r_{2}}\right)^{-1}=\frac{\delta V_{m} \cos \theta}{R_{0} T \ln \left(p_{g} / p_{l}\right)}$

where $V_{m}$ is the kelvin radius (m), $V_{m}$ is the molar volume of the water mist $\left(\mathrm{m}^{3} / \mathrm{mol}\right), \theta$ is the angle, taken here as $0^{\circ}, R_{0}$ is the universal gas constant $\left(\mathrm{J} \cdot \mathrm{K}^{-1} \cdot \mathrm{mol}^{-1}\right), T$ is the absolute temperature $(\mathrm{K}), p_{g}$ is the gas vapor pressure $(\mathrm{Pa}), p_{l}$ is the saturated vapor pressure of the water mist $(\mathrm{Pa})$, and $p_{g} / p_{l}$ is the relative humidity (dimensionless).

The negative pressure difference of the water membrane and the separation pressure are a pair of equilibrium forces, combined with Eqs. (8)-(10):

$\delta=\frac{H}{6 \pi L^{3}} \cdot \frac{\delta V_{m}}{R_{0} T \ln \left(p_{g} / p_{l}\right)}$

The thickness of the available water film is:

$L=\left[\frac{H}{6 \pi} \cdot \frac{V_{m}}{R_{0} T \ln \left(p_{g} / p_{l}\right)}\right]^{\frac{1}{3}}$

Under continuous spraying at $20^{\circ} \mathrm{C}$, the molar volume of water fog is $V_{m}=1.8 \times 10^{5} \mathrm{~m}^{3} / \mathrm{mol}$, the universal gas constant $R_{0}=8.31 \mathrm{~J} \cdot \mathrm{K}^{-1} \cdot \mathrm{mol}^{-1}$, the absolute temperature $T=293 \mathrm{~K}$, and the Hamack constant of water mist $H=3.72 \times 10^{-20} \mathrm{~J}$.

(2)Derivation and calculation of water film height

Water mist between the wires forms a water film under capillary action and presents a circular concave liquid surface under magnification. The height of the concave water film is affected by the total capillary force (negative pressure difference of the total water film) $F_{m}$, adjacent vibrating wire viscosity force $F_{v}$, and water mist gravity $F_{g}$.

According to the Young-Laplace equation [13], the capillary force (negative pressure difference of the water film) can be written as follows: 


$$
\Delta p=\frac{2 \delta}{r_{2}}=\frac{2 \delta \cos \theta}{r}
$$

As can be seen in Fig. 3,

$$
r_{2}=\frac{r}{\cos \theta}
$$

If the distance between the vibrating wire and steel wire is $d_{0}=2 r$, the total capillary force is as follows:

$$
F_{m}=2 \pi r \delta \cos \theta
$$

The capillary motion of the water mist between vibrating wire is unsteady and satisfies the Navier-Stokes equation. To simplify the calculation, the flow of water mist between the vibrating wire is regarded as steady flow, the radial and circumferential direction components of the flow velocity is set to 0 , and the flow is symmetrically distributed. The Navier-Stokes equation [20] can therefore be expressed by the special Hagen-Poiseuille equation [21]. The viscous force between vibrating wires can be obtained by the internal friction law of a Newtonian viscous fluid and the Hagen-Hagen-Poiseuille equation. The constitutive equations of Newtonian viscous fluid are as follows:

$$
\tau=\left.\mu \frac{d v}{d r_{0}}\right|_{r_{0}=r}
$$

where $\tau$ is the internal friction force on the wall of the vibrating wire $(\mathrm{Pa}), \mu$ is the viscosity of water mist $(\mathrm{Pa} \cdot \mathrm{S}), v$ is the incoming flow velocity $(\mathrm{m} / \mathrm{s})$, and $r_{0}$ is the radial coordinates (m).

The above formulations show that the viscous internal friction force of the water mist on the surface of the wire is linearly related to the velocity gradient, and the distribution law of the flow rate under the wind action is similar to that in a circular tube. Hagen-Poisuer's law can be used to express [19]:

$$
\frac{1}{r} \frac{d}{d r_{0}}\left(r_{0} \frac{d v}{d r_{0}}\right)=\frac{1}{\mu} \frac{\partial p}{\partial z}
$$

where $p$ is the water mist pressure $(\mathrm{Pa})$ and $z$ is the capillary tube length direction coordinate (m).

Taking into account the uniform change of the water film force between the vibrating wire, $\frac{\partial p}{\partial z}=\frac{\Delta p}{h^{1}}, h^{1}$ is the height of the water film $(\mathrm{mm})$ when it does not reach equilibrium. Integration of Eq. (17) with boundary conditions of $r_{0}=r, v=0 \mathrm{~m} / \mathrm{s}$, the distribution of water mist velocity on the cross section of vibrating wire is as follows, The derivation can be obtained:

$$
\frac{d v}{d r_{0}}=\frac{r_{0}}{2 \mu} \frac{\Delta p}{h^{1}}
$$

Because the velocity of the water mist varies along the radial direction between the wire, the average velocity is usually used to represent the changing velocity:

$$
\bar{v}=\frac{Q}{A}=\frac{\int_{0}^{r} 2 \pi r_{0} \nu d r_{0}}{\pi r^{2}}
$$


Eq. (18) is substituted into Eq. (19) to obtain:

$$
\frac{\Delta p}{h^{1}}=\frac{8 \mu \nu}{r^{2}}
$$

Replacement of Eq. (20) with Eq. (18) yields:

$$
\frac{d v}{d r_{0}}=\frac{r_{0}}{2 \mu} \cdot \frac{8 \mu \nu}{r^{2}}
$$

at $r_{0}=r$ :

$$
\left.\frac{d v}{d r_{0}}\right|_{r_{0}=r}=\frac{r}{2 \mu} \cdot \frac{8 \mu \bar{v}}{r^{2}}=\frac{4 \bar{v}}{r}
$$

The internal friction force on the surface of vibrating wire can be obtained by replacing Eq.

(22) with the Newtonian viscous fluid constitutive equation (16):

$$
\tau=\mu \cdot \frac{4 \bar{v}}{r}
$$

The viscous force of the adjacent vibrating wire is:

$$
F_{\mathrm{v}}=2 \pi r h \cdot \frac{4 \mu \bar{v}}{r}=8 \pi \mu \bar{v} h
$$

The gravity of the water mist between vibrating wires can be expressed as:

$$
F_{\mathrm{g}}=m g=\rho \pi r^{2} h g
$$

where $h$ is the rising water film height in the vibrating wire clearance $(\mathrm{mm})$ and $\rho$ is the water mist density $\left(\mathrm{kg} / \mathrm{m}^{3}\right)$.

Capillary action of the water film between the vibrating and steel wires is the result of the above three forces, according to the action direction of the force, $F_{m}=F_{v}+F_{g}$, namely:

$$
2 \pi r \delta \cos \theta=8 \pi \mu \bar{v} h+\rho g \pi r^{2} h
$$

The height of the capillary action of the water film in the gap of the vibrating wire is as follows:

$$
h=\frac{2 r \delta \cos \theta}{8 \mu \bar{v}+\rho g r^{2}}
$$

From Eq. (27), the rising height of water film capillarity depends on the distance between adjacent vibrating wires, contact angle between the water film and wire, surface tension of water film, and average flow velocity.

\section{Results and Discussion}

\section{Relationship between effective area of a water film on a vibrating wire and dust removal efficiency}

The dust filtration efficiency of a resonant chord grid depends on the uniform filling rate of the steel wire (Fig. 3). The filtered area (height $\times$ width) of the vibrating wire grid plate used in the experiments is $590 \times 550 \mathrm{~mm}$, the cylindrical steel wire diameter $d_{f}$ is $0.35 \mathrm{~mm}$, the steel wire spacing $d_{0}$ is $0.4 \mathrm{~mm}$, the filling rate $\beta_{s}$ is $46.70 \%$, and the total length of the 
double vibrating wire diameter is:

$$
L_{s}=\frac{8 \beta_{s} a b}{\pi d_{f}}
$$

where $L_{s}$ is the total diameter and length of the double vibrating wire (mm), $a$ is the length of single layer vibrating wire $(\mathrm{mm})$, and $b$ is the width of the single layer vibrating wire $(\mathrm{mm})$.

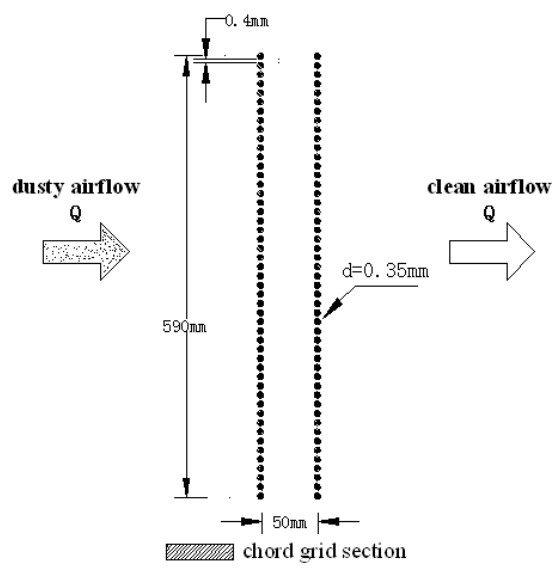

Fig. 3 Filling rate of the curvature of the resonant string grille

The working parameters are as follows: dust gas flow $Q\left(\mathrm{~m}^{3} / \mathrm{s}\right)$, dust concentration $C_{0}(\mathrm{~kg} / \mathrm{m})$ in front of the dust flow through the plate, dust concentration $C_{l}(\mathrm{~kg} / \mathrm{m})$ after dust flow through the plate, the dimensionless filtering efficiency $\eta_{1}$, the incoming flow velocity of the filtered gas is the velocity $v(\mathrm{~m} / \mathrm{s})$ of the wind flow at an infinite distance from the vibrating wire, and the velocity $\bar{v}(\mathrm{~m} / \mathrm{s})$ at the vibrating wire. The amount of dust collected by the vibrating wire per unit length $\phi(\mathrm{kg})$ is then:

$$
\begin{aligned}
& \phi=C_{0} \eta_{1} v d_{f} \\
& \eta_{1}=1-\frac{C_{1}}{C_{0}} \\
& v=\frac{v}{1-\beta_{s}}=\frac{Q}{a b\left(1-\beta_{s}\right)}
\end{aligned}
$$

For a vibrating wire dust catcher that is uniformly distributed in the resonant wire grid, a unit filter is assumed on the vibrating wire surface, and the dust concentration entering the unit is $C$. In a unit time, through the vibrating wire with a filter area of , the total number of dust particles in the filter unit is $d_{f} L_{s} \cdot \frac{d_{f} L_{s} d x}{\frac{\pi}{4} d^{2}{ }_{f} L_{s}}$. Therefore, the amount of dust particles captured by the filter unit per unit time is:

$$
-Q d C=d_{f} L_{s} \cdot \frac{d_{f} L_{s} d x}{\frac{\pi}{4} d^{2}{ }_{f} L_{s}} \cdot C \cdot A \cdot v \cdot \eta_{1}
$$

When Eq. (31) is substituted into Eq. (32), the following simplification and integral can be made:

$$
-\int_{C_{1}}^{C_{0}} \frac{d C}{C}=\int_{0}^{h} \frac{4 d_{f} L_{s} \eta_{1}}{\pi\left(1-\beta_{s}\right) d_{f}} d x
$$

The dust capture efficiency of simplified vibrating wire is: 
$\eta_{f}=1-\exp \left[-\frac{4 L_{s} h}{\pi\left(1-\beta_{s}\right)} \eta_{1}\right]$

For a vibrating wire under spray wetting condition, the trapping of dust particles by the steel wire and water film occurring between the strings is included in the process of dust particle capture. Under spraying conditions, the effective water film area between the vibrating wire can purify the dust.According to the formation process, the effective area of water film can be divided into effective areas of primary and secondary film formation. The effective area of primary film formation is that in which the water mist ejected from the nozzle forms between the wires. The effective area of secondary film formation forms under the combined action of turbulent buffeting and vortex shedding excitation. The secondary film-forming frequency is twice the fiber vibration frequency [22]. The experimental data show that the vibration frequency of the wire is the sum of the turbulent buffeting frequency and vortex shedding excitation frequency. The turbulent buffeting frequency is expressed by Eq. (35). As studied by Webster [23]. Under turbulent conditions, the relationship between the shedding frequency and vibrating wire Reynolds number is as follows:

$$
\begin{gathered}
f=\frac{v d_{f}}{d}\left[3.05\left(1-\frac{d_{f}}{d}\right)^{2}+0.28\right] \\
S r=0.198\left(1-\frac{19.7}{\mathrm{Re}}\right)
\end{gathered}
$$

Assuming that the thickness of the effective water film between the vibrating string steel wires is equal to the diameter of the vibrating string steel wire, the effective area function of the water film is expressed by the effective saturation of the water mist.

$$
A_{w}=[1+2(f+S r)]\left(1-\beta_{w s}\right) S_{w e} a b
$$

where $f$ is the dimensionless turbulent buffeting frequency, $S r$ is the dimensionless vortex shedding frequency, and $S_{w e}$ is the effective saturation of the water on the plate (dimensionless).

The effective saturation of water on the plate refers to water flowing in the gap, accounts for the volume of the entire plate gap, and is calculated as follows:

$$
S_{w e}=\frac{S_{w}-S_{w 0}}{1-S_{w 0}}
$$

Water saturation, which refers to the degree to which the grid gap is filled by water mist, is the water-to-void volume ratio and is related to spray pressure. Water saturation can be obtained from the experimental data concerning spray pressure. Part of the water in the gap does not flow owing to adsorption forces. This part of the water is called irreducible water. The irreducible water-to-wire void volume ratio is the irreducible saturation of water, which can be calculated by the static liquid holding capacity of the wire [17]. By replacing Eq. (38) with Eq. (34), the dedusting efficiency of the double-layer wet vibrating string steel wire is calculated as follows:

$$
\eta_{w f}=1-\exp \left[-\frac{4 L_{s} h}{\pi\left(1-\beta_{s}\right)} \eta_{1}\right]+[1+2(f+S r)]\left(1-\beta_{w s}\right) S_{w e}
$$

\section{Experimental determination and analysis of dust removal efficiency}

Eq. (39) shows that the dedusting efficiency increases proportionally to the effective area of the water film. The experimental results of the effective water film area and dust removal efficiency are shown in Table 3, and the specific behavior is shown in Fig. 4.

Table 3 Experimental results of effective water film area and dedusting efficiency 


\begin{tabular}{cccc}
\hline $\begin{array}{c}\text { spray pressure } \\
(\mathrm{MPa})\end{array}$ & $\begin{array}{c}\text { wind speed } \\
(\mathrm{m} / \mathrm{s})\end{array}$ & Effective area of water film $/ \mathrm{mm}^{2}$ & Dust removal efficiency/\% \\
0.3 & 3 & 0.29 & 86.30 \\
0.5 & 3 & 0.31 & 89.20 \\
0.7 & 3 & 0.35 & 92.69 \\
0.9 & 3 & 0.37 & 90.00 \\
1.1 & 3 & 0.38 & 89.12 \\
\hline
\end{tabular}

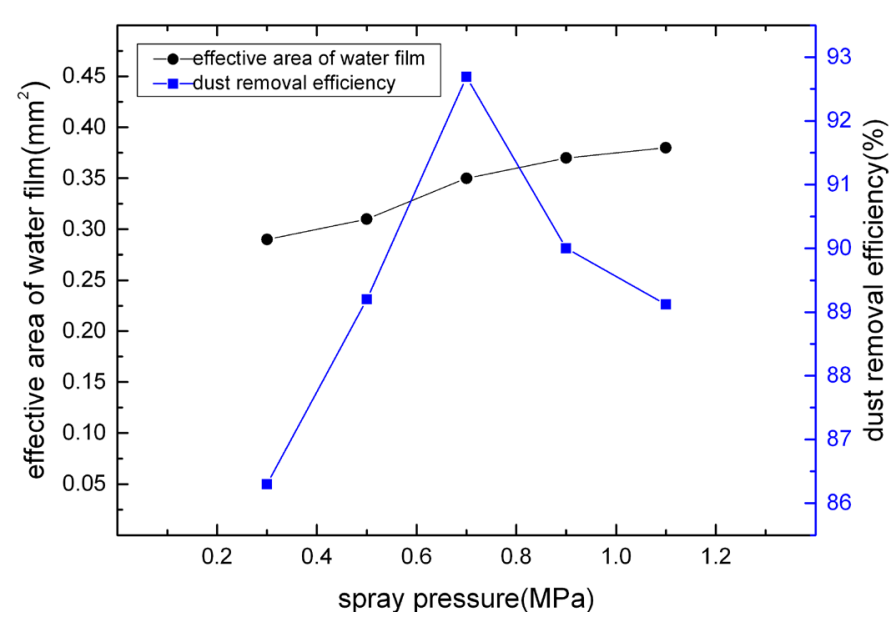

Fig. 4 Variable spray pressure effective water film area and dedusting efficiency

The theoretical value is consistent with the experimental measurements and shows that the dedusting efficiency is proportional to the effective water film area. A larger effective water film area is associated with higher dedusting efficiency. When the spray pressure changes from 0.3 to $0.5 \mathrm{MPa}$, the variation range of the dedusting efficiency is substantially larger than that of the effective water film area. When the water pressure is $>0.7 \mathrm{MPa}$, the effective water film area continues to increase whereas the dedusting efficiency decreases slightly, which shows that higher spray pressure is preferable but that there exists an optimal spray pressure. When the optimal spray pressure is obtained, it enhances water film formation, however, the film breaks more easily, which is not conducive to the improvement of dedusting efficiency.

\section{Conclusion}

(1) The adjacent steel wire is subjected to adhesion forces between the water mist and wire surface, resulting in the formation of a water film. Based on the Bond number, the water film formation conditions include wire spacing of $<0.34 \mathrm{~mm}$, which is consistent with the experimental results. According to mechanical equilibrium conditions, the negative pressure of the water film between the wires is equal to the separation pressure in the water film. Combined with the relationship between curvature radius of the film surface and relative humidity in the continuous spray environment, the film thickness can be derived from the Kelvin equation.

(2)The constitutive expression of Newtonian viscous fluid flowing along the wire under wind action is established and combined with the Navier-Stokes equation and Hagen-Poiseuille law. The expression of the height of the capillary action of the water film in the gap of steel wire is constructed. 
(3) Through experimental tests and theoretical analyses. The effective area of primary film formation forms between the gate wires by water mist ejected from the nozzles. The effective area of secondary film formation forms under the combined action of turbulent buffeting and vortex shedding vibration. The effective area function of the water film is expressed by the effective saturation of the water mist, and the dedusting efficiency of double-layer wet vibrating wire is calculated.

(4) From the expression of dedusting efficiency and experimental data, we conclude that the dedusting efficiency is proportional to the effective water film area. Larger effective water film area is associated with higher dedusting efficiency. When the dedusting wind speed is 3 $\mathrm{m} / \mathrm{s}$, the chord-grid distance ratio is 1.14 , the filling rate $\beta_{s}$ is $46.70 \%$, the optimum spray pressure is $0.7 \mathrm{MPa}$, the corresponding effective area of water film is $0.35 \mathrm{~mm}^{2}$, and the dedusting efficiency is $92.69 \%$.

\section{Acknowledgments}

We thank Esther Posner, PhD, from Liwen Bianji, Edanz Editing China (www.liwenbianji.cn/ac), for editing the English text of a draft of this manuscript.

\section{References}

[1] Fairs, Lowrie G(1958)High Efficiency Fibre Filters for the Treatment of Fine Mists . Trans. Instn. Chem. Engrs 36:476-485.

[2] Agranovski I E, Braddock R D(1998)Filtration of Liquid Aerosols on wettable fibrous filters. AICHE Journal 44(12):2775-2783.

[3] Jakub M. Gac(2015)A Simple Numerical Model of Pressure Drop Dynamics During the Filtration of Liquid Aerosols on Fibrous Filters. Separation Science and Technology 50(13):2015-2022.

[4] Jakub M. Gac, Anna Jackiewicz, Łukasz Werner, Szymon Jakubiak(2016)Consecutive filtration of solid particles and droplets in fibrous filters"Separation and Purification Technology 170(10): 234-240.

[5] Ryan Mead-Hunter, Andrew J. C. King, Gerhard Kasper,Benjamin J. Mullins(2013)Computational fluid dynamics (CFD) simulation of liquid aerosol coalescing filters.Journal of Aerosol Science 61(11):3649.

[6] Gong L H(2002)Theoretical and Experimental study on Dust removal Technology of Wet vibrating Fiber Grid.Shenyang: Northeast University.

[7] TAN C, JIANG Z A(2015)Experimental study on dust-control by water curtain in underground mines. Journal of China University of Mining \& Technology 44(6):1002-1007.

[8] Jin L Z, Li J P, Su Y F, et al(2010)Theory of Mine Dust Prevention and Control.Beijing: Science Press.

[9] Li Y C, Zhang Y H, Xiong S S(2011)Study on dedusting technology with high-Pressure atomizing and vibrating fiber grid.Nonferrous Metals(Mining Section) 63(1):58-61.

[10] Wu C F, Li Y J, Chen Z Y, et al(2017)Experimental study on influencing factors of dust removal performance for dust collector with charged water mist vibration fiber grid"Journal of Safety Science and Technology 13(3):162-168. 
[11] Yuan T, Chen Z, Liu W J, et al(2014)Application of Capillary Mechanics to Separation Process of Superhydrophilic Membrane and Its Mechanical Model.Journal of South China University of Technology(Natural Science Edition) 42(10):82-89.

[12] Gao S Q, Liu H P(2010)Capillary Mechanics.Beijing: Science Press.

[13] Lin N, Li G L,Wang H D, et al(2015)Research Progress of Surface Free Energy's Computing Methods and the Influence on the Properties of Material Surface.Materials Review 29(11):30-35+40.

[14] A. Alexandrou Himonas, Dionyssios Mantzavinos, Fangchi Yan(2019)The nonlinear Schrödinger equation on the half-line with Neumann boundary conditions. Applied Numerical Mathematics 141(7):2-18.

[15] Zhuang D W, Yang Y F, Hu H T, et al(2016) Visualization and prediction model on shape of liquid bridge.CIESC Journal 67(6):2224-2229.

[16] Ronald F. Probmtein, Dai G C, Fang G N, et al(1992)Introduction to Physicochemical Fluid Dynamic.Shanghai:East China Institute of Chemical Technology Press.

[17] Gu H Y(2002)Study on Mechanism and Experiment of Dust Filtration about Wet Fiber Layer.Qingdao:Qingdao University of Technology.

[18] Kentaro Tanaka, Katsumi Iwamoto(2016)Effective Shear Displacement on Lateral Adhesion Force of a Liquid Bridge Between Separated Plates.Tribology Letters 64 (1):1-7.

[19] A W.Adamson, A P. Gast(1997)Physical Chemistry of Surfaces, 6th edition.New York: Wiley Interscience Press.

[20] Zhang X X, Zhang Z T, Zhang W F, et al(2018)Comparison of five eddy viscosity turbulence models in numerical simulation of flow over a two-dimensional square cylinder.Acta Aerodynamica Sinica 36(2): 339-349.

[21] Chen X J, Yao G Q(2017)An improved model for permeability estimation in low permeable porous media based on fractal geometry and modified Hagen-Poiseuille flow. Fuel 210(12):748-757.

[22] Gong L H, Wang Y M, Liu T M, et al(2003)Research on dust removing mechanism and efficiency of wet vibration fibrous grid.Journal of Safety and Environment 13(3):32-35.

[23] Webster S, McBride R, Barton J, et a1(1992)Air flow measurement by vortex shedding from multimode and monomode optical fibers.Mess Sci Techno 13(2):210-216. 
Figures

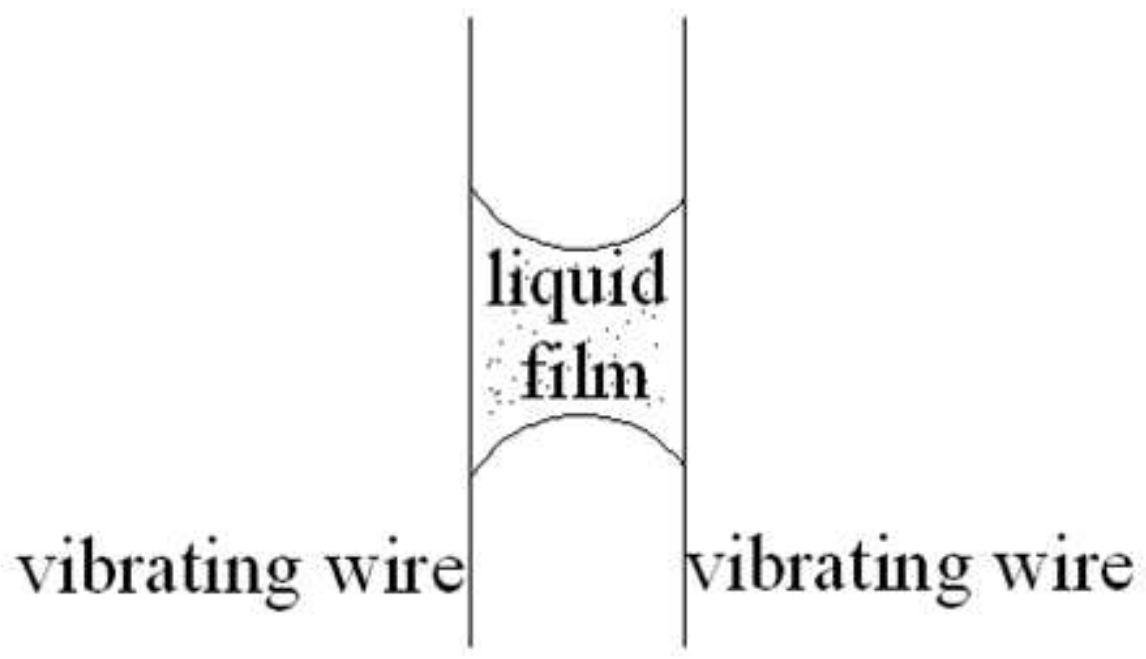

Figure 1

Concave water film surface between vibrating wire strings

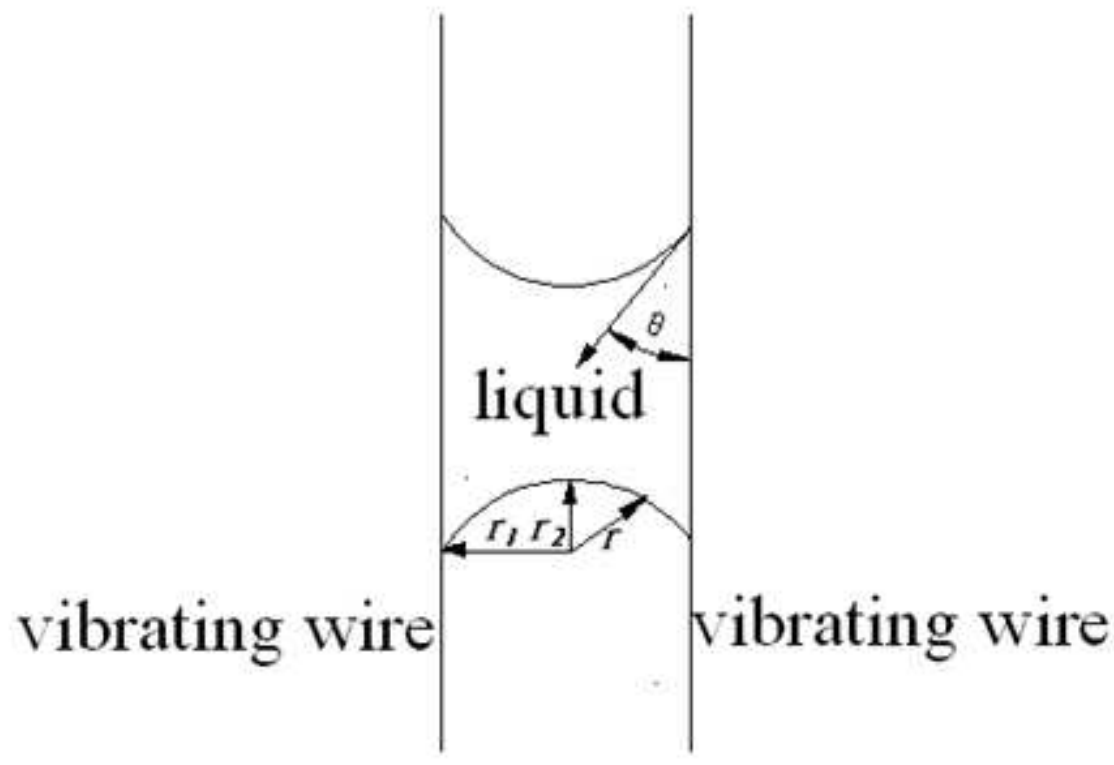

Figure 2

Radius of the concave liquid surface 


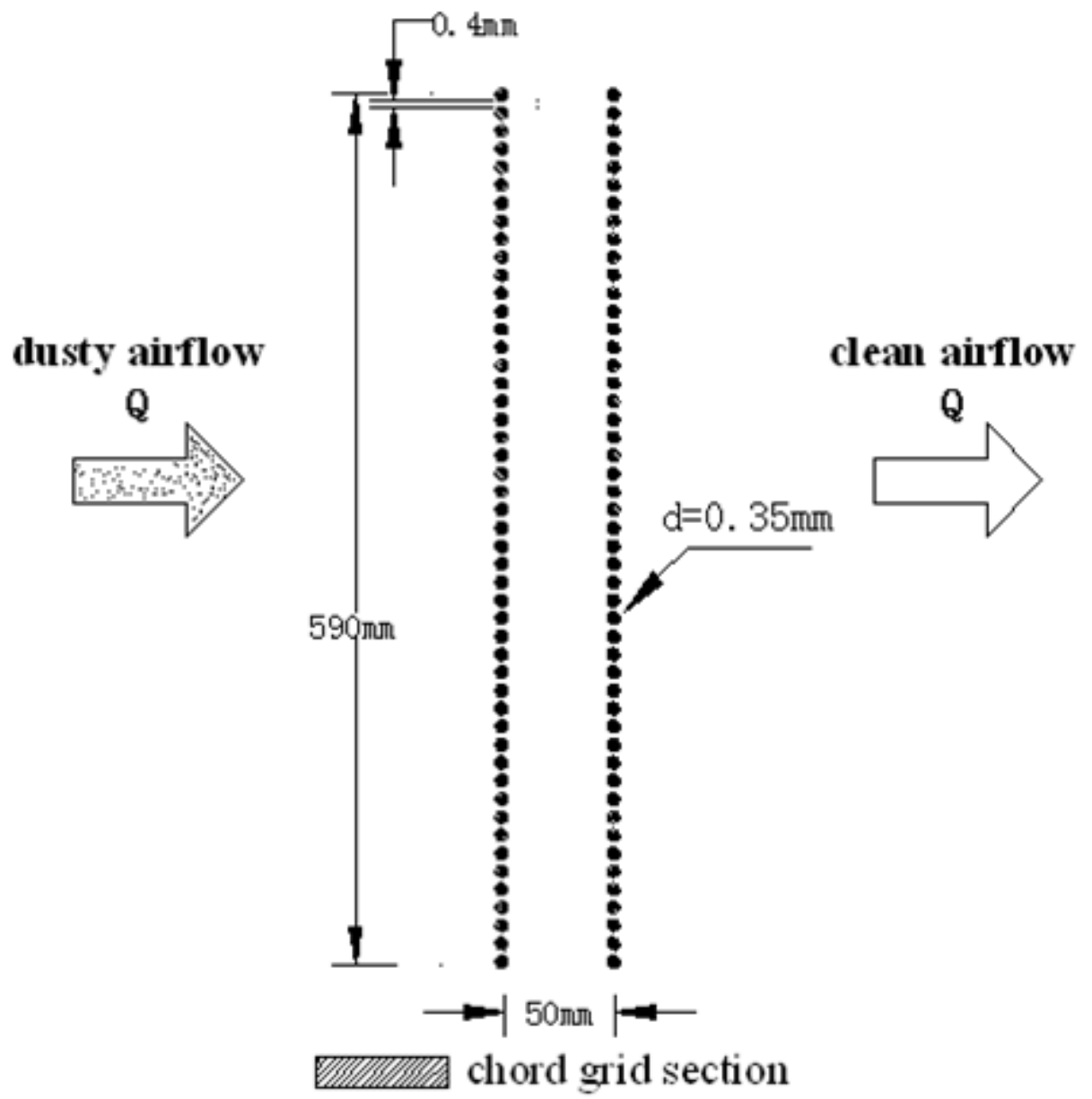

Figure 3

Filling rate of the curvature of the resonant string grille 


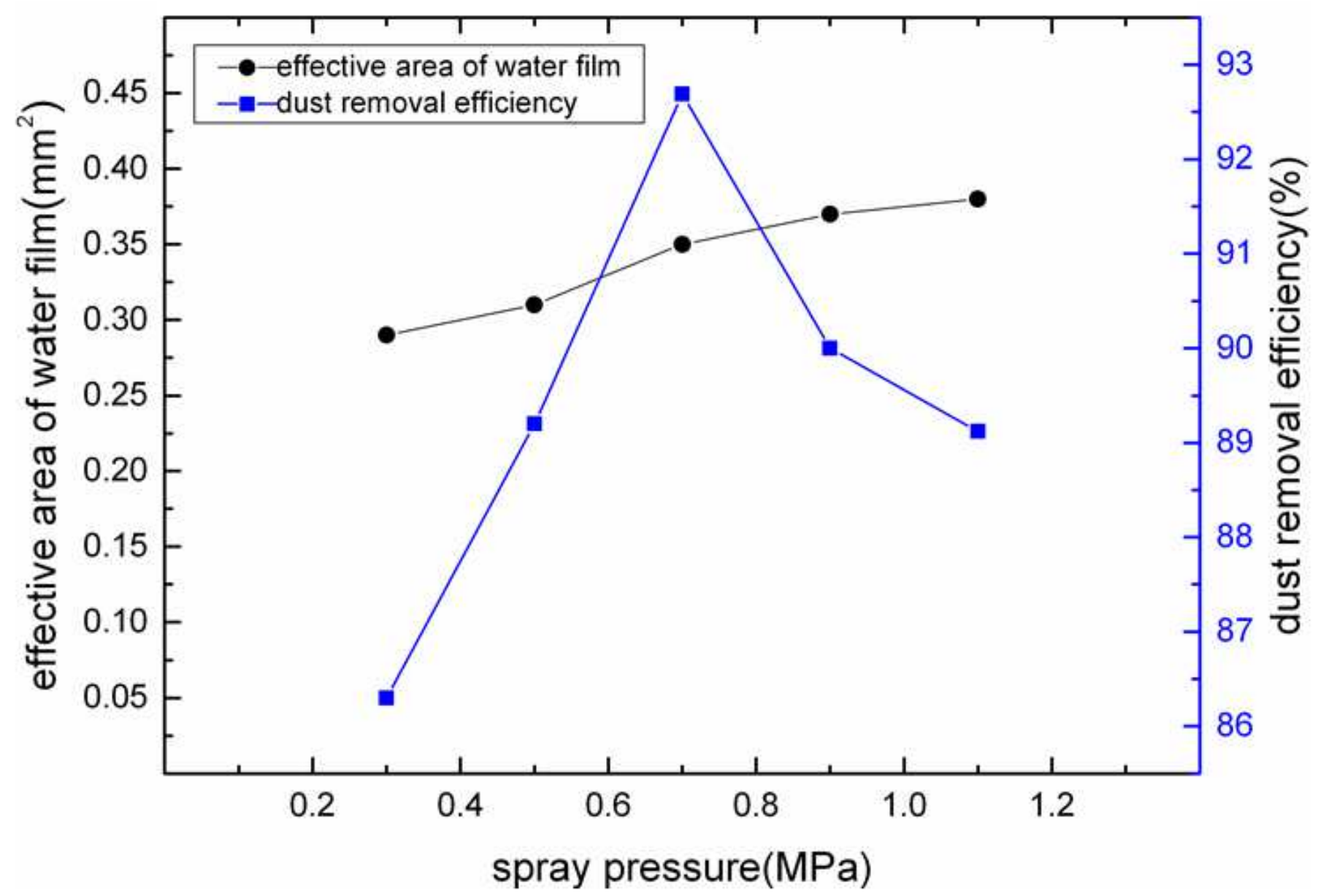

Figure 4

Variable spray pressure effective water film area and dedusting efficiency 